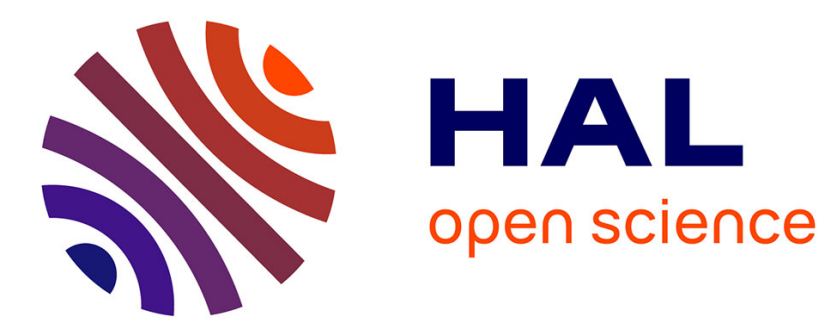

\title{
Voluntary head movement and allocentric perception of space \\ Mark Wexler
}

\section{- To cite this version:}

Mark Wexler. Voluntary head movement and allocentric perception of space. 2002. hal-00000021

\section{HAL Id: hal-00000021 \\ https://hal.science/hal-00000021}

Preprint submitted on 10 Oct 2002

HAL is a multi-disciplinary open access archive for the deposit and dissemination of scientific research documents, whether they are published or not. The documents may come from teaching and research institutions in France or abroad, or from public or private research centers.
L'archive ouverte pluridisciplinaire HAL, est destinée au dépôt et à la diffusion de documents scientifiques de niveau recherche, publiés ou non, émanant des établissements d'enseignement et de recherche français ou étrangers, des laboratoires publics ou privés. 


\title{
VOLUNTARY HEAD MOVEMENT AND ALLOCENTRIC PERCEPTION OF SPACE
}

\author{
MARK WEXLER
}

\begin{abstract}
Although visual input is egocentric, some visual perceptions and representations may be allocentric, i.e., independent of the observer's vantage point or motion. By comparing the visual perception of $3 \mathrm{D}$ object motion during voluntary and involuntary motion in human subjects, results of three experiments show that the motor command contributes to the objective perception of space: observers executing voluntary head movements are more likely to apply, consciously and unconsciously, spatial criteria relative to an allocentric frame of reference than while undergoing similar involuntary displacements - which lead to a more egocentric bias. Furthermore, details of the motor command are crucial to spatial vision, since allocentric bias decreases or disappears unless self-motion and motor command match.
\end{abstract}

An important property of our visual system is that its viewpoint constantly moves through space, usually as a result of voluntary motor action on the observer's part. At least two reference frames are therefore possible for spatial vision: an egocentric or subjective frame centered on the eye and therefore fixed to the head, and an allocentric or objective, earth-fixed frame.

Perceiving spatial information in an allocentric frame is perhaps the ultimate form of spatial constancy, and has important advantages. An ecological advantage is that it allows one to see whether an object is moving at all, rather than moving relative to the observer. There are also computational advantages: the world can be assumed to be stable in this reference frame, and representations of objects and spatial relations do not have to be updated as one moves about. However, retinal data are in an egocentric frame - they contain information only on relative motion between object and observer. To convert this to an allocentric frame, self-motion-the movement of eyes through space as the result of eye rotations and head movements - must be evaluated and added to relative motion.

Does spatial vision make use of information about self-motion, and, in particular about head motion? (The rest of this article, which focuses on $3 \mathrm{D}$ vision, will concentrate on head movements - which result in depth information from motion parallax - whereas eye rotations do not.) One way to to address this question is to compare the visual performance of observers in different self-motion conditions, while keeping visual input constant. Held and his colleagues compared actively moving to passively moved human observers, finding effects of voluntary action on visuomotor learning [1] (and on visual development in cats [2]). Though comparisons between actively moving and immobile observers had seemed to show no effect of self-motion on

Date: September 15, 2002. Submitted to Psychological Science. 
the precision of spatial vision [3, it was recently demonstrated that actively generated motion parallax can lead to different perceptions of 3D shape than the same visual stimulation experienced by an immobile observer [4. The complement to the perception of $3 \mathrm{D}$ shape and motion of objects is the perception of self-motion relative to the environment; it has been shown that extra-retinal information about head and eye movements is integrated with optic flow in the perception of heading [5, 6].

The use of allocentric reference frames has been studied mainly in the context of spatial memory, where it could constitute a basis for the formation of mental maps that allow for correct spatial behavior, independent of an animal's position or orientation [7]. In the hippocampus of rats, for instance, 'place cells' code position in an allocentric frame [8. In monkey hippocampus, neurons have recently been found that code allocentric position during active, but not passive, motion [9]; in monkey posterior parietal cortex, there is a hierarchy of cells that code positions in retinotopic, craniotopic, and spatiotopic (allocentric) frames [10. In human infants, active displacement has been found to favor spatial memory in an allocentric frame [11], and a similar result has been obtained in a robotic learning model [12].

These neurophysiological and behavioral studies have pointed to voluntary self-motion playing a role in long-term, learning processes in spatial perception and representation. The experiments presented here compare immediate visual perception in subjects either engaged in voluntary motion, or passively undergoing similar movements. The results show for the first time an immediate effect of voluntary self-motion on spatial vision, and notably on the capacity to use an allocentric reference frame. Two experimental paradigms are used: one in which the choice of reference frame is an explicit and conscious part of the task (Experiment 1), and one in which it is unconscious (Experiments 2 and 3).

\section{EXPERIMENT 1}

While performing backward-and-forward head movements, subjects viewed a binocularly presented virtual object, also in motion. Their task was to decide whether the object moved, with respect to the otherwise invisible room, in the same or in the opposite direction as they did - an explicitly allocentric criterion (cf. ref. [13]). As illustrated in Fig. 1, the object's movement was yoked to that of the subject with a gain $\gamma$ : when the subject's head underwent a displacement $\Delta z$ perpendicular to the image, the object moved by $\gamma \Delta z$ along the same axis. Thus, if the point of subjective equality (where half the answers are "towards" and half are "away") falls at $\gamma_{0}=0$, subjects do make use of an allocentric stationarity criterion, as instructed; if, on the other hand, $\gamma_{0}=1$, an egocentric criterion is used.

The main independent variable of interest was the type of movement: in one block (VOL), the subject performed voluntary backward-and-forward head movements while seated in an immobilized wheelchair; in the other block (INVOL), the subject's head was fixed to the back of the wheelchair, which was moved by the experimenter. To ensure that head trajectories in the two conditions were similar, trials were automatically aborted and restarted unless trajectories met certain kinematic criteria. 


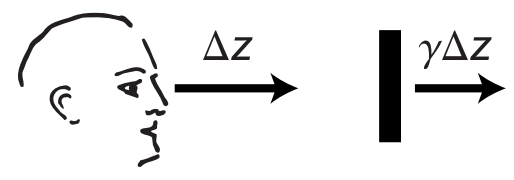

FiguRE 1. In Exp. 1, subjects engaged in voluntary and involuntary back-and-forth head movements; for each displacement $\Delta z$ of the midpoint between the subject's eyes towards or away from it, the virtual object, projected with binocular disparity, underwent the same translation (in an earth-fixed, allocentric reference frame) as the subject's head, but with a gain along the $z$-axis, $\gamma \Delta z$.

\section{Methods.}

Participants. Eleven paid volunteers participated, with 6 performing the VOL block first, and 5 the INVOL block first.

Apparatus. Head position and orientation were measured with a high-precision head tracker. Stimuli displayed on a computer monitor coupled to shutter glasses to provide binocular disparity. Other than the stimuli, all experiments were performed in complete darkness. In particular, the monitor frame was invisible. Further details are described in ref. [14].

Stimuli. The virtual object projected on the monitor was a $5 \times 5$ square grid parallel to the plane of the monitor, with each cell $1 \mathrm{~cm}$ in length and edges horizontal and vertical. The initial position of the grid center was $\left(x_{0}, y_{0}, Z\right)$, with $Z=-10,0$, or $10 \mathrm{~cm}$, and $x_{0}, y_{0}$ the initial coordinates of the midpoint between the subject's eyes. (In these coordinates, the $x y$-plane is the monitor with the origin at the center, the $x$-axis points to the subject's right, the $y$-axis upwards, and the $z$-axis towards the subject.) When the subject's head moved by $(\Delta x, \Delta y, \Delta z)$ between two monitor refreshes, the grid translated by $(\Delta x, \Delta y, \gamma \Delta z)$, where $\gamma$ varied from -1.1 to +1.1 from trial to trial. The grid center thus always remained directly opposite the point between the subject's eyes. The stimulus was viewed binocularly, because without cues to absolute depth, concomittant motion could be interpreted as illusory depth.

Procedure. In the vOL condition, the subject sat in an immobilized wheelchair; a trial began when the subject's position (midpoint between the eyes) was within $5 \mathrm{~cm}$ of the point $100 \mathrm{~cm}$ from the monitor screen, directly opposite its center. The subject performed oscillatory head movement along the $z$-axis (perpendicular to the monitor). Subjects were instructed to minimize lateral and vertical movement (indeed, the mean RMS displacements in the $x y$ plane did not exceed $28 \%$ of those along the $z$-axis, for any subject in any experiment). The starting direction (forward or backward) was verbally cued. When displacement along the $z$-axis reached $6 \mathrm{~cm}$, a tone sounded to instruct the subject to change direction; if the displacement exceeded $12 \mathrm{~cm}$, the trial was aborted and re-started. The subject performed 2.5 such cycles, with the stimulus grid visible only after the first half-cycle. The subject 


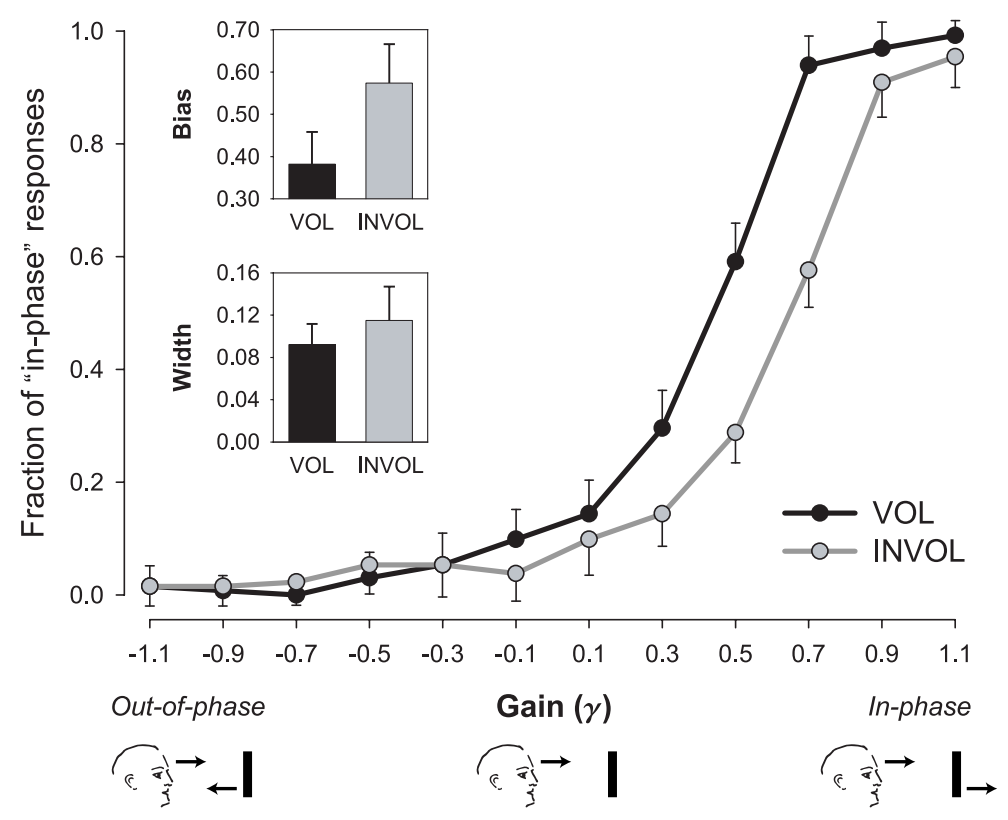

Figure 2. Results of Exp. 1. Curves indicate responses averaged over subjects. Mean bias and width were calculated by fitting individual subject data with logistic curves, and averaging the parameters thus obtained. Bars indicate between-subject standard errors.

could then stop moving; the stimulus was replaced by two icons representing "same" and "opposite direction" responses, from which the subject selected by pressing a mouse button.

In the INVOL condition, the subject's head was attached to the back of the wheelchair. With the brakes removed, the experimenter, standing behind the wheelchair, moved it backward-and-forward (the wheelchair was modified so that it could not turn sideways), with the subject's head motion (as measured by the headtracker) subject to the same kinematic restrictions as in the corresponding VOL trials.

Results and discussion. The raw data are shown in Fig. 2 as the fraction of "same-direction" responses for different values of $\gamma$. Individual subject data were fitted to the logistic function $\left[1+3^{-\left(\gamma-\gamma_{0}\right) / w}\right]^{-1}$, where the bias $\gamma_{0}$ indicates the point of subjective stationarity, and the width $w$ the precision. Mean biases and widths are shown in Fig. 2. In vol, the mean point of subjective stationarity was closer $\left(\gamma_{0}=0.38\right)$ to the allocentric criterion $\left(\gamma_{0}=0\right)$ than in INVOL $\left(\gamma_{0}=0.57, t_{10}=4.59, p<0.001\right)$. The widths, on the other hand, were not significantly different in VOL and INVOL (0.092 and 0.115 , respectively, $\left.t_{10}=1.00\right)$. To make sure that learning effects were not confounded with the VOL/INVOL variable, one group of subjects performed the conditions in the order VOL-INVOL, while the other group did so in the opposite order; there was no significant interaction between the group and the VOL/INVOL variables. 
To properly compare performance in VOL and INVOL one has to make sure that head trajectories were very similar in these conditions, and that any small differences in the trajectories could not account for the performance differences. This analysis is presented in the Appendix.

\section{EXPERIMENT 2}

Experiment 1 shows that when using an allocentric criterion is explicitly required, subjects are capable of doing so to a higher degree when actively moving than when passively undergoing similar trajectories. Exp. 2 investigates whether the same is true when the use of an allocentric frame is an unconscious part of the task, using an ambiguous stimulus.

To resolve ambiguities of movement, the visual system often 'attempts', unconsciously, to minimize motion [15, 16, 17, 18. Recently, we have devised an ambiguous stimulus for the moving observer in which one solution minimizes motion in an allocentric frame, while the other does so in an egocentric frame [14. Studying observers in voluntary motion, we found a bias towards the allocentric solution. What will happen to this allocentric bias in the case of involuntary motion?

Here, we use the same stimulus as in the second expriment of ref. [14] see Figure 3. Concretely, while the subject performs voluntary (VOL) or involuntary (INVOL) backwards-and-forwards head movements as in Exp. 1 , a set of moving dots is displayed on the computer screen. The dots' movement is yoked to that of the subject in such a way as to allow the two 3D interpretations described above (see Fig. 3b): a plane $(A)$ whose center is stationary in an allocentric frame, or a plane $(E)$ whose center undergoes the same movement as the subject, and is therefore stationary in an egocentric frame. The two planes also rotate in the image plane, but about axes that differ by $90^{\circ}$; the subject's task, following the presentation of the stimulus, is to indicate its perceived axis of rotation. From this response (which is cognitively opaque), we can determine which solution the subject unconsciously chooses - and how the degree of allocentric bias depends on the subject's motion being voluntary.

\section{Methods.}

Participants. Twelve new subjects participated, with each performing 2 VOL blocks and 1 INVOL block, in the order VOL-INVOL-VOL.

Apparatus. Same as Exp. 1, except with the stimulus presented monocularly.

Stimuli. A set of pixels was presented monocularly, the projections of 50 dots on a virtual plane with slant $\sigma=45^{\circ}$ and tilt chosen from the set $0^{\circ}, 30^{\circ}, \ldots, 330^{\circ}$. On the average, the size of the stimulus was $10^{\circ}$. The density of the dots was circularly symmetric (to minimize texture depth cues), and proportional to $r^{-4 / 3}$, with $r$ the distance from the center: lower density at the edges made them irregular and less salient. The virtual object rotated about a frontal axis perpendicular to its normal, with angular speed $v / \tan \sigma$, where $v$ was the subject's speed along the $z$-axis normalized by the distance from the stimulus - so that the two maxima of rigidity were the $A$ and $E$ solutions described above [14]. 


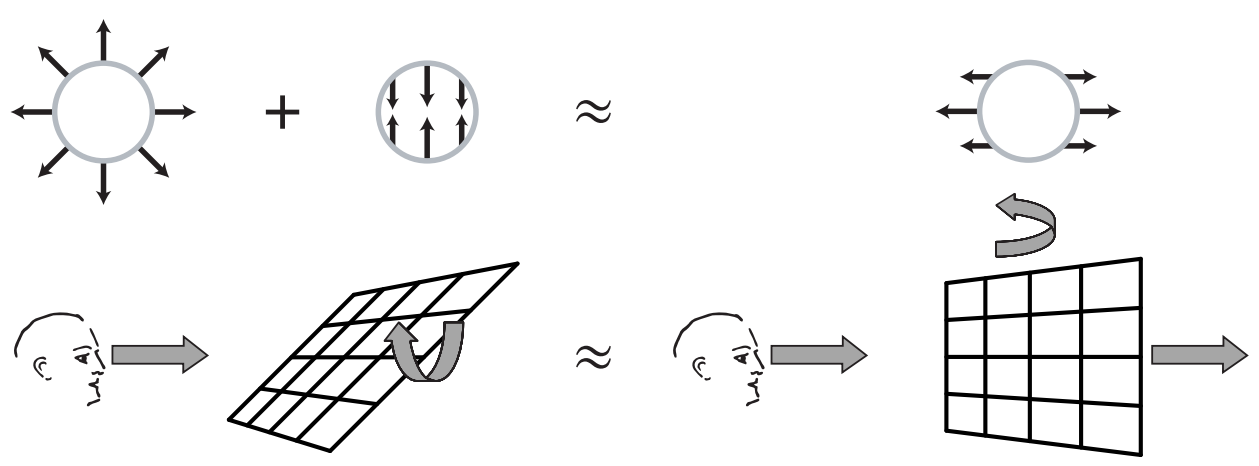

(a)

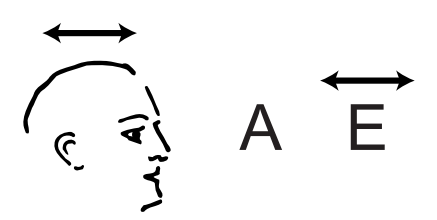

(b)

Figure 3. (a) Experiments 2 and 3 relied on an ambiguity in optic flow. During an observer's translatory motion towards or away from a plane whose center is fixed (in an earth-fixed, allocentric frame), and which rotates with a certain speed about an axis perpendicular to its normal, the optic flow is roughly the same as a plane whose center is fixed with respect to the moving subject (i.e., which reproduces the subject's translatory movements), and which rotates at the same speed as the first plane, but whose orientation and axis of rotation are shifted by $90^{\circ}$ with respect to those of the first plane. (b) Thus, the moving observer can perceive a $3 \mathrm{D}$ configuration that minimizes motion in an allocentric frame (A), or one that does so in an egocentric frame (E). For further details, including a mathematical derivation, see ref. [14].

Procedure. Similar to Exp. 1, with minor changes (1.5 cycles of motion, starting distance of $90 \pm 10 \mathrm{~cm}$, amplitude $7 \mathrm{~cm}$, and a limitation of the period of oscillation to below $6 \mathrm{~s}$ ). During the response phase, a probe line could be rotated by inclining a joystick. Subjects adjusted the probe so that it appeared to coincide with the rotation axis of the stimulus (or its projection).

Results and discussion. The distributions of axis angle responses, with respect to the axis of the $A$ solution, are shown in Fig. 4. There is a clear difference between the conditions: subjects chose the $A$ solution more often in VOL than in INVOL. To quantify this difference, the allocentricity is defined as the fraction of trials in which responses were closer to the $A$ than to the $E$ solution. Allocentricity, also shown in Fig. 4, was significantly higher 
Exp. 2
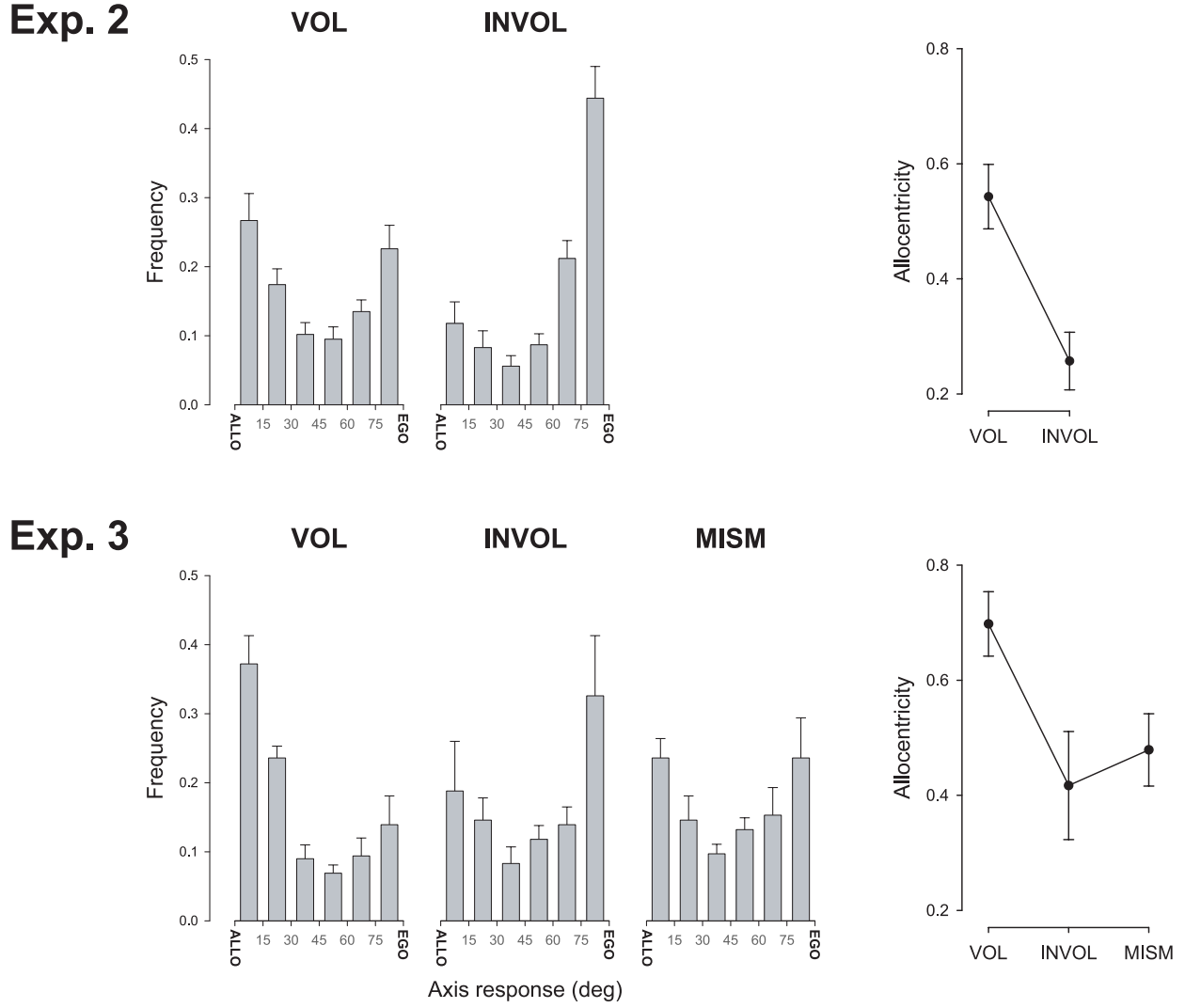

Figure 4. Results of Exps. 2 and 3. Histograms represent distributions of absolute axis angle differences with respect to the allocentrically most stationary solution. Curves show the allocentricity measure, the fraction of responses closer to the solution that minimizes motion in an allocentric reference frame. Bars represent between-subject standard errors.

in VOL $(0.543)$ that in INVOL $(0.257)\left(t_{11}=6.8, p<10^{-4}\right)$. This VOLINVOL difference was not due to any learning or sequence effects: $t$ tests show no significant difference in allocentricity between the first and second VOL blocks. As shown in the Appendix, trajectories in VOL and INVOL are very similar, and the small differences do not account for differences in performance. These results show that when spatial reference frame criteria are unconscious, rather than explicit, as in Exp. 1, subjects also make use of an allocentric reference frame more during voluntary than involuntary motion.

\section{EXPERIMENT 3}

The results of Exp. 2 do not tell us whether any voluntary action resulting in the movement of the eye through space is sufficient to trigger a bias towards an allocentric least-motion criterion (the trigger hypothesis); or whether, in the active observer, the details of the motor command lead to a predicted self-motion, which is used to compensate egocentric sensory 
data and to convert it to an allocentric frame (the prediction hypothesis). To distinguish between these hypotheses, a mismatch condition (MISM) was added to Exp. 2, in which observers actively generated self-motion by turning the wheels of a wheelchair with their arms. Since none of the observers had had any experience with this type of locomotion, resulting self-motion did not match the details of the the motor command. The trigger hypothesis predicts that the results of the MISM condition should resemble those in the VOL condition, while according to the prediction hypothesis results of MISM should resemble those in INVOL.

Methods. Identical to Exp. 2, except for the additional MISM condition, in which the subject's head was attached as in INVOL, but the motion was driven by the subject's turning the wheelchair wheels with his or her arms. Six new subjects participated (none with prior experience of arm-driven locomotion in a wheelchair, and none needing more than a few minutes to learn to operate the wheelchair with the required kinematics), with half performing the conditions in the order VOL-INVOL-MISM-VOL, and half in the order VOL-MISM-INVOL-VOL.

Results and discussion. Results are shown in Fig. 4. As in Exp. 2, subjects had a significantly higher allocentricity in VOL than in INVOL $\left(t_{5}=4.97\right.$, $p<0.01$ ). (The subjects in Exp. 3 had higher mean allocetricity than those in Exp. 2, but these between-group differences were not significant. On the other hand, the VOL-INVOL differences were very similar in the two groups: 0.286 in Exp. 2, 0.281 in Exp. 3.) Subjects had a significantly higher allocentricity in VOL than in MISM $\left(t_{5}=3.61, p<0.05\right)$, while there was no significant difference between INVOL and MISM $\left(t_{5}=1.12, p=0.3\right)$. Thus, although it is impossible to exclude the contribution of voluntary arm motion in the mismatch condition, we can conclude that it is insufficient to account for the degree of allocentric bias during voluntary motion. These results therefore agree with the prediction hypothesis, and contradict the trigger hypothesis. We conclude that in voluntary motion, the detailed motor command is converted into a metric prediction of self-motion (unless there is a mismatch), which is then used to convert visual data to an allocentric frame.

\section{General Discussion}

The results of three experiments show, for the first time, that voluntary motion can play an important role in the immediate visual perception of $3 \mathrm{D}$ object motion and shape, and notably in the use of allocentric spatial criteria. Experiment 1 shows that when the task involves conscious allocentric criteria (i.e., the explicit judgment of motion in an allocentric frame), subjects are better able to apply such criteria when actively generating their head movements than when passively undergoing similar trajectories. Experiment 2 shows that a similar result holds in a case where allocentric criteria are unconscious: subjects are more likely to apply least-motion criteria in perceptually disambiguating 3D motion and structure in an allocentric frame in voluntary than in involuntary motion. Finally, Experiment 3 shows that in the case of voluntary motion, the details of the motor command play 
an important role: a mismatched motor command leads to similar performance as in involuntary motion.

Extra-retinal self-motion information can be categorized into two types, motor and proprioceptive. Motor information arises from the origin of the motor signal in the brain, an 're-afferent copy' or corollary discharge of which are used in vision. [19, 20]. Proprioceptive information arises from sensory feedback once the subject is moving, in particular from vestibular and somatosensory signals. The main difference between the voluntary and involuntary motion is that, while proprioceptive signals are available in both types of motion, motor signals are absent or highly diminished in involuntary motion. However, in the present experiments some secondary somatosensory signals also differed between voluntary and involuntary conditions, and vestibular signals may be different in the two cases [21, 22]. However, if the robust differences between voluntary and involuntary motion demonstrated here are due to motor signals, one can conclude that motor signals in head motion play an important role in the allocentric perception of space. Furthermore, from the results of Exp. 3, we can conclude that it is not the mere presence of a corollary discharge, but the details of the motor command that are crucial to spatial vision. Insofar as vision in an allocentric frame is a form - perhaps the ultimate form - of spatial constancy, this finding echoes the role of efference copy in spatial constancy in the context of eye movements [23, 24, 25, 26, 27].

What role could learning play in the incorporation of self-motion signals in spatial vision? For instance, would the results of the mismatch condition be different in wheelchair-bound subjects used to this form of locomotion? Could subjects learn to make do with nothing but proprioception with sufficient exposure to passive locomotion? The last proposition seems doubtful, given the existing prevalence of passive motion (e.g., in automobiles) in our culture. Held and Freeman [1] found that visuo-motor learning took place in both voluntary and involuntary locomotion, but proceeded faster in the voluntary case. In light of these questions, it would be useful to examine how the effects found in the present study evolve with learning.

The experiments presented here provide psychophysical evidence that human spatial vision uses an allocentric reference frame more often in voluntary self-motion and an egocentric frame more often in the involuntary case, that this is so whether the reference frame is a conscious or unconscious part of the subject's task, and that the voluntary-involuntary difference diminishes or disappears unless there is a detailed match between the motor command and the observer's displacement. These results show that not only the observer's self-motion cannot be disregarded in spatial vision, but that the observer's active role in initiating and producing that motion is crucial.

\section{APPENDIX}

In comparing the visual perception performance across movement conditions in all three experiments, a crucial question is: to what extent are any movement trajectory differences responsible for the effects observed? By 'trajectories,' we will mean the translations of the eye(s) during the portion of each trial in which the stimulus was visible. The crucial, $z$-components of 

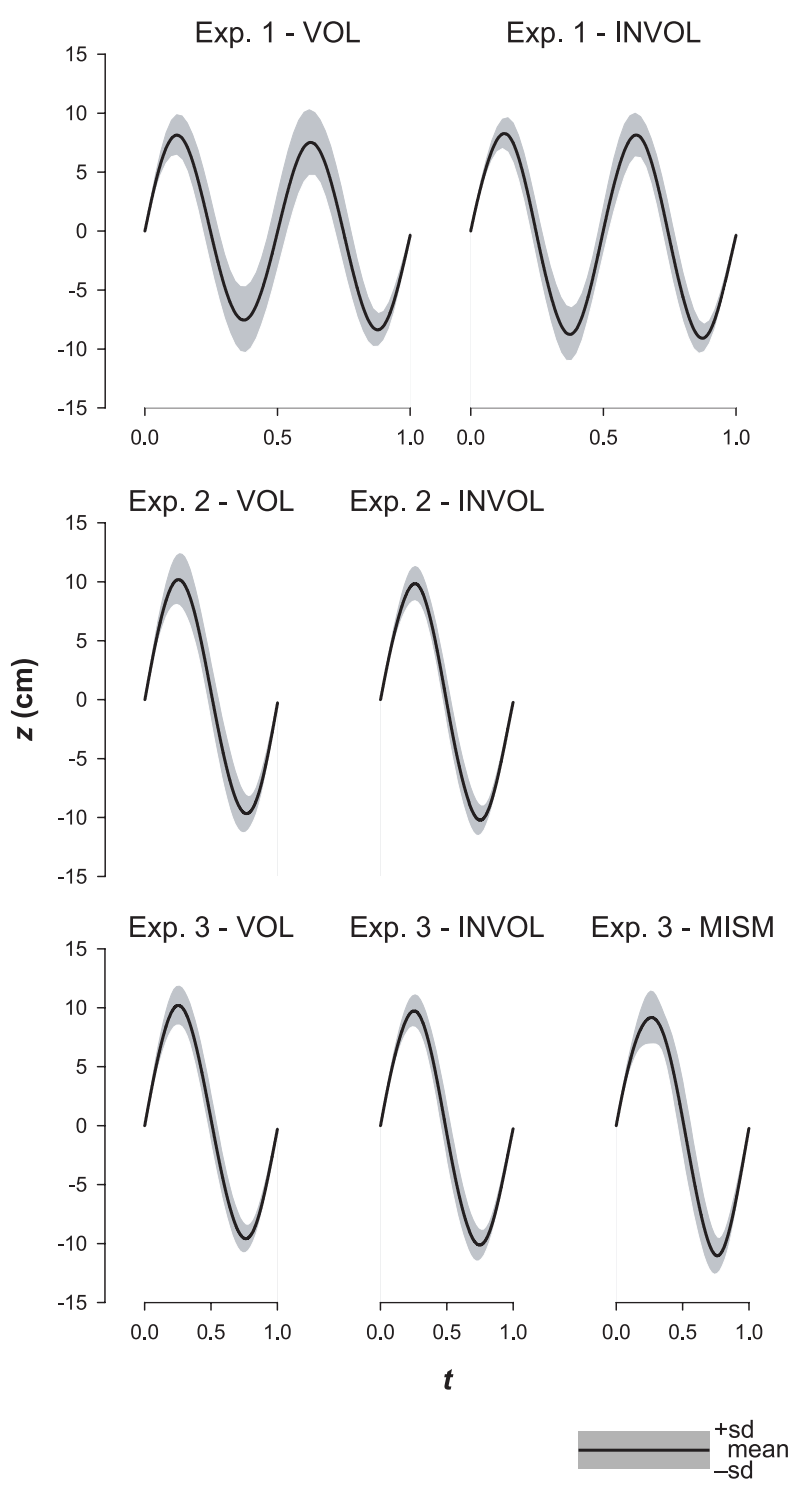

FiguRE 5. Average eye displacements along the $z$-axis, normalized by trial duration, for all subjects in all conditions of all three experiments, during the portion of each trial for which the stimulus was visible (2 cycles in Exp. 1, 1 cycle in Exps. 2 and 3. Gray bars represent standard deviation.

the trajectories are shown in Fig. 5, which qualitatively demonstrates that trajectories were very similar across conditions in each experiment. Three types of quantitative analyses have been carried out, and are summarized below. The results of these analyses, for all three experiments, are that (1) trajectory differences are minor, and (2) such trajectory differences as exist between the conditions cannot account for the effects observed.

Trajectory variations. One way of defining the difference between trajectories in two conditions is to compare variations between conditions to those 
within conditions. To define a measure inverse to variation, we use the mean correlation between $z$-displacements in all pairs of trials; to calculate correlations between unequal-length trials, the $z$-coordinate was resampled in 100 time bins in each trial. For example, in Exp. 1, the mean within-condition correlation is 0.867 in VOL, 0.935 in INVOL, while the mean correlation between VOL and INVOL is 0.894 . This means that, while trajectory variations in INVOL are lowest (understandable, since the same experimenter always produced those trajectories), the differences between trajectories in VOL and INVOL are lower than the variations within the VOL condition, and approximately equal to average variations within the two conditions. Similar results hold for Exps. 2 and 3.

Homogenized trajectories. In order to test whether the small differences in trajectory kinematics could be responsible for the performance differences between the conditions, trials were subdivided into narrow, homogeneous subsets by various kinematics pareameters. In Exp. 1, for example, the RMS $z$-speed of $87 \%$ of trials in VOL and INVOL fell between 15 and 28 $\mathrm{cm} / \mathrm{s}$. These trials were divided into 13 equal bins, each bin $1 \mathrm{~cm} / \mathrm{s}$ wide and each having a nearly uniform speed distribution. Within every bin, bias in VOL was lower (closer to zero) than in INVOL: a non-parametric test for difference in bias between VOL and INVOL would therefore yield $p<0.05$. A similar analysis for $z$-displacements yielded a similar result, and so did corresponding analyses for allocentricities in Exps. 2 and 3. Thus, when we compare across conditions only those subsets of trials in which the distributions of a given kinematic parameter are nearly identical, we find the same effects as when we include all trials. Therefore, any variations in kinematic parameters are unlikely to be responsible for the differences in visual performance between conditions.

No correlations between trajectories and performance. Finally, as an additional analysis to test for any dependance of responses on trajectory kinematics, the following quantities were regressed against the axis angle responses in Exps. 2 and 3, within each block for each subject: trial duration, RMS of $z$-displacements, RMS of $x y$-displacements, RMS of $z$-speeds, and RMS of $z$-accelerations. After performing t tests on Fisher-transformed correlation coefficients over all subjects for each quantity in each block, none of the kinematic quantities was found to have any significant effect on responses, in any condition ( $p>0.05$, Bonferoni adjusted). Thus, despite the small differences between trajectory kinematics in VOL and INVOL, it is reasonable to compare responses in these conditions, since the responses did not depend in any significant or consistent way on the kinematics.

\section{ACKNOWLEDGEMENTS}

This work was partly supported by the CNRS and by a grant from Essilor International. Thanks to Jacques Droulez, Ivan Lamouret and Jeroen van Boxtel for discussions. 


\section{REFERENCES}

[1] R. Held and D.J. Freedman. Plasticity in human sensorimotor control. Science, 142:455-462, 1963.

[2] R. Held and A. Hein. Movement-produced stimulation in the development of visually guided behavior. Journal of Comparative and Physiological Psychology, 56:872-876, 1963.

[3] B. Rogers and M. Graham. Motion parallax as an independent cue for depth perception. Perception, 8(2):125-134, 1979.

[4] M. Wexler, F. Panerai, I. Lamouret, and J. Droulez. Self-motion and the perception of stationary objects. Nature, 409:85-88, 2001.

[5] C. S. Royden, M. S. Banks, and J. A. Crowell. The perception of heading during eye movements. Nature, 360(6404):583-585, 1992.

[6] J.A. Crowell, M.S. Banks, K.V. Shenoy, and R.A. Andersen. Visual self-motion perception during head turns. Nature Neuroscience, 1:732-737, 1998.

[7] E.C. Tolman. Cognitive maps in rats and man. Psychological Review, 55:189-208, 1948.

[8] J. O'Keefe and L. Nadel. The hippocampus as a cognitive map. Oxford, 1978.

[9] H. Nishijo, T. Ono, S. Eifuku, and R. Tamura. The relationship between monkey hippocampus place-related neural activity and action in space. Neuroscience Letters, 226:57-60, 1997.

[10] L.H. Snyder, K.L. Grieve, P. Brotchie, and R.A. Andersen. Separate body- and worldreferenced representations of visual space in parietal cortex. Nature, 394:887-891, 1998.

[11] L. P. Acredolo, A. Adams, and S. W. Goodwyn. The role of self-produced movement and visual tracking in infant spatial orientation. J Exp Child Psychol, 38(2):312-327, 1984.

[12] K. Hiraki, A. Sashima, and S. Phillips. From egocentric to allocentric spatial behavior: A computational model of spatial development. Adaptive Behavior, 6:371-391, 1998.

[13] H. Wallach, L. Stanton, and D. Becker. The compensation for movement-produced changes in object orientation. Perception and Psychophysics, 15:339-343, 1974.

[14] M. Wexler, I. Lamouret, and J. Droulez. The stationarity hypothesis: an allocentric criterion in visual perception. Vision Research, 41:3023-3037, 2001.

[15] M. Wertheimer. Experimentelle Studien über das Sehen von Bewegung. Zeitschrift für Psychologie, 61:161-265, 1912.

[16] H. Wallach. Über visuell wahrgenommene Bewegungsrichtung. Psychologische Forschung, 20:325-380, 1935.

[17] H. Wallach and D.N. O'Connell. The kinetic depth effect. Journal of Experimental Psychology, 45:205-217, 1953.

[18] S. Ullman. The interpretation of visual motion. MIT Press, Cambridge, Mass., 1979.

[19] R.W. Sperry. Neural basis of the spontaneous optokinetic response produced by visual inversion. Journal of Comparative and Physiological Psychology, 43:482-489, 1950.

[20] E. von Holst and H. Mittelstaedt. Das reafferenzprinzip. Naturwissenschaften, 37:464476, 1950.

[21] R. A. McCrea, G. T. Gdowski, R. Boyle, and T. Belton. Firing behavior of vestibular neurons during active and passive head movements: vestibulo-spinal and other noneye-movement related neurons. Journal of Neurophysiology, 82(1):416-428, 1999.

[22] J. E. Roy and K. E. Cullen. Selective processing of vestibular reafference during self-generated head motion. Journal of Neuroscience, 21:2131-2142, 2001.

[23] J.K. Stevens, R.C. Emerson, G.L. Gerstein, T. Kallos, G.R. Neufeld, C.W. Nichols, and A.C. Rosenquist. Paralysis of the awake human: Visual perceptions. Vision Research, 16(1):93-98, 1976.

[24] L. Stark and B. Bridgeman. Role of corollary discharge in space constancy. Perception and Psychophysics, 34(4):371-380, 1983.

[25] B.L. Guthrie, J.D. Porter, and D.L. Sparks. Corollary discharge provides accurate eye position information to the oculomotor system. Science, 221(4616):1193-1195, 1983. 
[26] J.R. Duhamel, C.L. Colby, and M.E. Goldberg. The updating of the representation of visual space in parietal cortex by intended eye movements. Science, 255(5040):90-92, 1992.

[27] M.A. Sommer and R.H. Wurtz. A pathway in primate brain for internal monitoring of movements. Science, 296(5572):1480-1482, 2002.

LPPA, Collège de France, 11 Pl. Marcelin Berthelot, 75005 Paris, France E-mail address: wexler@ccr.jussieu.fr

$U R L:$ http://wexler.free.fr/ 